\title{
Correlation studies of fission-fragment neutron multiplicities
}

\author{
M. Albertsson, ${ }^{1}$ B. G. Carlsson, ${ }^{1}$ T. Døssing, ${ }^{2}$ P. Möller, ${ }^{1,3}$ J. Randrup $\odot,{ }^{4}$ and S. Åberg $\odot^{1, *}$ \\ ${ }^{1}$ Mathematical Physics, Lund University, S-221 00 Lund, Sweden \\ ${ }^{2}$ Niels Bohr Institute, University of Copenhagen, 2100 Copenhagen $\emptyset$, Denmark \\ ${ }^{3}$ P. Moller Scientific Computing and Graphics, P.O. Box 75009, Honolulu, Hawaii 96836, USA \\ ${ }^{4}$ Nuclear Science Division, Lawrence Berkeley National Laboratory, Berkeley, California 94720, USA
}

(Received 19 May 2020; revised 2 November 2020; accepted 8 December 2020; published 19 January 2021)

\begin{abstract}
We calculate neutron multiplicities from fission fragments with specified mass numbers for events having a specified total fragment kinetic energy. The shape evolution from the initial compound nucleus to the scission configurations is obtained with the METROPOLIS walk method on the five-dimensional potential-energy landscape, calculated with the macroscopic-microscopic method for the three-quadratic-surface shape family. Shape-dependent microscopic level densities are used to guide the random walk, to partition the intrinsic excitation energy between the two proto-fragments at scission, and to determine the number of neutrons evaporated from the fragments. The contribution to the total excitation energy of the resulting fragments from statistical excitation and shape distortion at scission is studied. Good agreement is obtained with available experimental data on neutron multiplicities in correlation with fission fragments from ${ }^{235} \mathrm{U}\left(n_{\text {th }}, f\right)$. With increasing neutron energy a superlong fission mode grows increasingly prominent, which affects the dependence of the observables on the total fragment kinetic energy.
\end{abstract}

DOI: 10.1103/PhysRevC.103.014609

\section{INTRODUCTION}

A long-standing challenge in nuclear fission is the dependence of the average neutron multiplicity $\bar{v}$ on the fragment mass number $A$. The characteristic sawtooth behavior of $\bar{v}(A)$ is well illustrated for ${ }^{235} \mathrm{U}\left(n_{\mathrm{th}}, f\right)$; see, e.g., Ref. [1]. Because the number of neutrons evaporated is indicative of the excitation energy in the emitting fragment, it is of key importance to understand the degree of excitation of the fission fragments, as a function of $A$.

To a good approximation, the total excitation energy of a given fission fragment is the sum of two distinct contributions. One is the share of total statistical excitation received by the distorted proto-fragment at the time of scission. The other contribution results from the relaxation of the fragment shape from its distorted form at scission to its equilibrium shape which converts the change in potential energy into additional fragment heat. Different theoretical descriptions of the fission process [2-7] have yielded different results for the amount of distortion energy. Furthermore, because it is the sum of the two contributions that determines the energy available for

*Corresponding author: sven.aberg@matfys.lth.se

Published by the American Physical Society under the terms of the Creative Commons Attribution 4.0 International license. Further distribution of this work must maintain attribution to the author $(s)$ and the published article's title, journal citation, and DOI. Funded by Bibsam. neutron evaporation, it is difficult to determine the individual contributions from the measured $\bar{v}(A)$ alone.

However, by studying the dependence of $\bar{v}(A)$ on the total fragment kinetic energy (TKE) it may be possible to gain important insight into how the fission-fragment excitation energy is composed. Such correlation measurements were performed recently, yielding $\bar{v}\left(A\right.$; TKE) for ${ }^{235} \mathrm{U}\left(n_{\mathrm{th}}, f\right)$ [8]. In this paper, we discuss how variations of TKE are associated with structure-dependent variations in the contributions to the fission-fragment excitations, leading in turn to observable variations of the TKE-constrained neutron multiplicity, $\bar{v}(A ; \mathrm{TKE})$.

Due to the highly dissipative character of collective nuclear dynamics, it has proven possible to model the shape evolution of a fissioning nucleus as a METROPOLIS walk on the multidimensional potential-energy surface [9-11]. By using shape-dependent microscopic level densities [12] for guiding the shape evolution, a consistent framework was obtained for calculating the energy-dependent fission-fragment mass distribution [13].

Recently, shape-dependent microscopic level densities were employed also for the calculation of the excitation energy partition between the fission fragments [7]. In that treatment, it was assumed that the statistical excitation energy available at scission is divided microcanonically between the two proto-fragments whose distorted shapes later on relax to their ground-state forms. It was found [7] that this treatment leads to a reasonably good reproduction of $\bar{v}(A)$ measured for ${ }^{235} \mathrm{U}(n, f)$ at both thermal energies and $E_{n}=5.55 \mathrm{MeV}$ [14], in particular for asymmetric fission events. The study brought out the important influence of the specific structure of 
the various proto-fragments whose level densities affect the energy partitioning significantly.

We now go further and study the energy dependence of the structure effects by gating on specific values of TKE. In such a more detailed study, the specification of a particular TKE value selects the total excitation energy (TXE). The resulting fragment excitation energies can then be calculated and the associated mean neutron multiplicities, $\bar{v}(A$; TKE), can be obtained.

Thus, for the first time, a fission model based on microscopic level densities, combined with a five-dimensional potential-energy surface obtained with the macroscopicmicroscopic method, is applied to calculate more complex correlations between observables, namely, the average neutron multiplicity from fission fragments of given $A$ for events with a particular TKE. A phenomenological deterministic model of prompt neutron emission was recently applied to the same problem, yielding very good agreement with data [17].

The method of the calculation is briefly presented in Sec. II. In Sec. III we discuss the total kinetic energy as well as contributions from intrinsic and distortion energy at scission to the excitation energies of the primary fission fragments. In Sec. IV the results for the neutron multiplicities are presented, where the role of the superlong (SL) fission mode is particularly studied. Finally, Sec. V presents a summary and a discussion.

\section{METHOD OF CALCULATION}

The calculations closely follow Ref. [7]. The evolution of the nuclear shape from the ground-state shape to scission is treated as a METROPOLIS random walk on the potentialenergy landscape $U(\chi)$ [9]. The shape $\chi$ is described by the three-quadratic-surface (3QS) parametrization $[15,16]$ which has five parameters: the overall elongation given by the quadrupole moment $q_{2}$, the neck radius $c$, the spheroidal deformations $\varepsilon_{\mathrm{f} 1}$ and $\varepsilon_{\mathrm{f} 2}$ of the endcaps of the two nascent fragments, and the mass asymmetry $\alpha$. For each of the more than $6 \times 10^{6}$ nuclear shapes considered, the microscopic level density is calculated by the combinatorial method [12] up to about $6 \mathrm{MeV}$ of excitation energy and is extrapolated to higher energies using the calculated shell and pairing energies [13].

The initial configuration is assumed to be a compound nucleus having the excitation energy $E_{0}^{*}=E_{n}+S_{n}$, where $E_{n}$ is the kinetic energy of the incoming neutron being absorbed and $S_{n}$ is the corresponding neutron separation energy. With $M_{0}$ as the mass of the compound nucleus, the total energy is given by $E_{\mathrm{tot}}=M_{0}+E_{0}^{*}$ which is conserved during the subsequent evolution. Consequently, at a given shape $\chi$ the local intrinsic energy is given by $E^{*}(\chi)=E_{\text {tot }}-U(\chi)$ because the shape motion is assumed to be so strongly damped that the local collective kinetic energy is negligible.

In the considered fission reaction ${ }^{235} \mathrm{U}(n, f)$ the compound nucleus ${ }^{236} \mathrm{U}$ can have either angular momentum $I=3$ or 4 . The angular momentum is conserved in the METROPOLIS random walk by considering level densities with a fixed angular momentum for each shape. Since $I=3$ and 4 give very similar results [13] the presented calculations are performed at $I=4$.
As in our earlier work [13], the shape changes are selected by the METROPOLIS method using the associated shapedependent microscopic level densities $\rho(\chi)$, ensuring detailed balance, $P\left(\chi \rightarrow \chi^{\prime}\right) / P\left(\chi^{\prime} \rightarrow \chi\right)=\rho\left(\chi^{\prime}\right) / \rho(\chi)$.

The asymmetry $\alpha$ is assumed to be frozen in when the neck radius has shrunk to $c=c_{0}=2.5$ fm [7,13]. Subsequently, the system reaches a scission configuration at $c=$ $c_{\mathrm{sc}}=1.5 \mathrm{fm}$ where the shapes of the proto-fragments are determined (see Sec. IIID), and the available intrinsic energy is partitioned between them (see Sec. III C). The choice of $c_{\mathrm{sc}}=1.5 \mathrm{fm}$ is discussed in Sec. III B. The initially distorted proto-fragments are being accelerated by their mutual Coulomb repulsion and the shapes eventually revert to equilibrium forms. Their original distortion energies are thereby converted to additional intrinsic excitation. Subsequently, after full acceleration has been achieved, each excited primary fragment evaporates neutrons as long as it is energetically possible (see Sec. IV).

For each reaction case considered, a total of $10^{6}$ fission events are generated and for each one we record the mass numbers of the two primary fission fragments, $A_{\mathrm{L}}$ and $A_{\mathrm{H}}$, their total kinetic energy TKE, as well as the number of neutrons evaporated from each one, $v_{\mathrm{L}}$ and $v_{\mathrm{H}}$, as would be done in an ideal experiment.

\section{ENERGIES IN THE FISSION PROCESS}

In Sec. III A we introduce the various key energies, namely, the TXE and the corresponding TKE, as well as the decomposition of the individual proto-fragment excitations into intrinsic and distortion energies. The calculated TKE values are discussed and compared to experimental data in Sec. III B. Then, in Sec. III C we describe how the intrinsic energy available at scission is partitioned between the two proto-fragments and how this depends on TKE. Finally, the variation of the distortion energy with TKE and fragment mass is analyzed in Sec. III D.

\section{A. Key energy quantities}

Once the initial compound nucleus has been prepared, we follow an ensemble of shape evolutions, as described in Sec. II. These represent possible evolutions of the fissioning system subject to the conservation of the total energy $E_{\text {tot }}$. When the evolving system has attained its scission shape, $\boldsymbol{\chi}_{\mathrm{sc}}$, it is assumed to divide into two distorted and excited protofragments which subsequently recede and accelerate while their shapes gradually relax to their equilibrium forms.

We assume that the strongly damped description of the shape evolution remains valid until scission, so the proto-fragments are formed with vanishing kinetic energy. Furthermore, the fragments typically carry several $(\approx 6)$ units of angular momentum, but we ignore the associated rotational energy, which is relatively small, $\approx 1-2 \mathrm{MeV}$. The combined intrinsic excitation energy of the two proto-fragments at scission is then given by the corresponding local intrinsic energy:

$$
E_{\mathrm{L}}^{\mathrm{intr}}+E_{\mathrm{H}}^{\mathrm{intr}}=E^{*}\left(\chi_{\mathrm{sc}}\right)=E_{\mathrm{tot}}-U\left(\chi_{\mathrm{sc}}\right) .
$$


The partitioning of the intrinsic energy among the two fragments is assumed to be statistical (see Sec. III C).

For a given mass partition, $A_{0} \rightarrow A_{\mathrm{L}}+A_{\mathrm{H}}$, the $Q$ value is $Q_{\mathrm{LH}}^{*}=M_{0}+E_{0}^{*}-M_{\mathrm{L}}-M_{\mathrm{H}}$. This quantity represents the energy available for the total fragment kinetic energy, TKE, and the combined excitation energies of the two primary fragments, TXE, after the acceleration and the shape relaxation have been completed:

$$
Q_{\mathrm{LH}}^{*}=\mathrm{TKE}+E_{\mathrm{L}}^{*}+E_{\mathrm{H}}^{*}=\mathrm{TKE}+\mathrm{TXE} .
$$

In the present paper, we assume that the proto-fragments have spheroidal shapes with eccentricities equal to those of the corresponding endcaps of the $3 \mathrm{QS}$ scission shape, $\varepsilon_{\mathrm{L}, \mathrm{sc}}=$ $\varepsilon_{\mathrm{fl}}\left(\chi_{\mathrm{sc}}\right)$ and $\varepsilon_{\mathrm{H}, \mathrm{sc}}=\varepsilon_{\mathrm{f} 2}\left(\chi_{\mathrm{sc}}\right)$. The distortion energy of protofragment $i=\mathrm{L}, \mathrm{H}$ can then be expressed as $E_{i}^{\text {dist }}=U_{i}\left(\varepsilon_{i, \mathrm{sc}}\right)-$ $U_{i}\left(\varepsilon_{i, \mathrm{gs}}\right)$, where $U_{i}(\varepsilon)$ denotes the potential energy of deformation of the fragment (see Sec. IIID). As the proto-fragments gradually attain their ground-state shapes, their distortion energies are being converted into additional intrinsic excitation energy, so the final excitation energy is the sum of the original intrinsic energy at scission and the distortion energy:

$$
E_{\mathrm{L}}^{*}=E_{\mathrm{L}}^{\text {intr }}+E_{\mathrm{L}}^{\text {dist }}, \quad E_{\mathrm{H}}^{*}=E_{\mathrm{H}}^{\text {intr }}+E_{\mathrm{H}}^{\text {dist }} .
$$

Thus, with $\mathrm{TXE}=E_{\mathrm{L}}^{*}+E_{\mathrm{H}}^{*}$, the total kinetic energy is determined from Eq. (2), TKE $=Q_{\mathrm{LH}}^{*}-\mathrm{TXE}$.

\section{B. Total kinetic energy}

The dependence of the average TKE on the heavyfragment mass number is shown in Fig. 1(a) for ${ }^{235} \mathrm{U}\left(n_{\mathrm{th}}, f\right)$. Two different scission scenarios are considered. The first scenario uses $c_{\mathrm{sc}}=2.5 \mathrm{fm}$, the neck radius $c_{0}$ at which the mass division is assumed to freeze out. The corresponding scission configurations are relatively compact and the resulting TKE values exceed the experimental data significantly for all divisions. This suggests that the fledging fragments maintain contact for a while after their masses have been determined. Therefore, as in our previous study [7], we assume that the effective loss of contact occurs later on when the neck radius has shrunk further to $c_{\mathrm{sc}}=1.5 \mathrm{fm}$, The corresponding shapes are then more elongated and, consequently, the resulting TKE values are lowered. Furthermore, the internal excitation energies are higher and the emerging proto-fragments have larger quadrupole moments.

In the region beyond $A_{\mathrm{H}} \geqslant 132$, where the average TKE exhibits a steady decrease with $A_{\mathrm{H}}$, the experimental data are very well reproduced by the calculations. However, in the more symmetric region, the extracted TKE values exceed the measured values considerably, by up to $20 \mathrm{MeV}$. This discrepancy may be due to the extraordinary elongation of the associated scission shapes which may not be adequately described within the three-quadratic-surface shape family in terms of which the potential-energy surface has been tabulated. An insufficient elongation leads an overprediction of TKE and an underestimate of the statistical excitation. As a consequence, the neutron multiplicity from fission fragments in the symmetric region will be too low by up to one neutron per fragment, as is seen in Fig. 3 of Ref. [7].

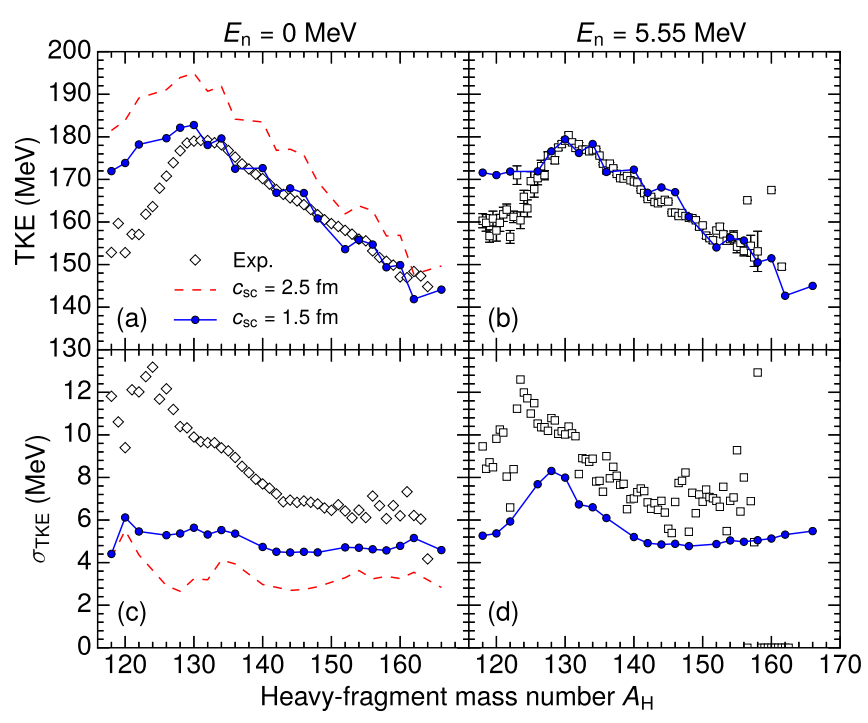

FIG. 1. Panel (a) shows the average total fragment kinetic energy TKE vs the heavy-fragment mass number $A_{\mathrm{H}}$ for ${ }^{235} \mathrm{U}\left(n_{\mathrm{th}}, f\right)$ for two values of the scission neck radius, $c_{\mathrm{sc}}=1.5 \mathrm{fm}$ (filled circles connected by solid blue lines) and $c_{\mathrm{sc}}=2.5 \mathrm{fm}$ (dashed red line). In (c) the calculated width of the TKE distribution, $\sigma_{\mathrm{TKE}}$, is shown for the same two values of $c_{\mathrm{sc}}$. Panels (b) and (d) are similar to (a) and (c), but are for a higher incident neutron energy, $E_{n}=5.55 \mathrm{MeV}$, and only results for the adopted scission radius, $c_{\mathrm{sc}}=1.5 \mathrm{fm}$, are shown. Measured values of TKE are shown for thermal fission [18] (open diamonds) and for $E_{n}=5.55 \mathrm{MeV}$ [14] (open squares).

As seen in Fig. 1(c), the width of the TKE distribution for a given $A_{\mathrm{H}}$ is underestimated in the calculations for both scission conditions, but the agreement with data is generally better for the adopted value, $c_{\mathrm{sc}}=1.5 \mathrm{fm}$, than for $c_{\mathrm{sc}}=2.5$ $\mathrm{fm}$. For the employed value, the underestimation is about $50 \%$ in the region of symmetric fission and decreases with increasing fission-fragment asymmetry. The underestimation may (at least in part) be due to the fact that the calculations include only even-even fragment pairs having (approximately) equal $N / Z$ ratios, namely, that of ${ }^{236} \mathrm{U}$. As a consequence of this restriction, there is only one $(N, Z)$ combination for a given $A$, whereas the actual fission process populates several combinations and thus leads to a broader TKE distribution.

Figures 1(b) and 1(d) show the average TKE and the width $\sigma_{\mathrm{TKE}}$, respectively, versus the heavy-fragment mass number for incoming neutrons of energy $E_{n}=5.55 \mathrm{MeV}$. The measured TKE values are very well reproduced not only for $A_{\mathrm{H}} \geqslant$ 132, as was the case for thermal fission [see Fig. 1(a)], but even down to $A_{\mathrm{H}}=126$, leaving only a rather narrow region around symmetry with a significant overestimate, by up to $10 \mathrm{MeV}$.

Also the calculated widths agree better with data for energetic than for thermal neutrons [Fig. 1(d)], though the calculated values generally are too small. Furthermore, the data exhibits a maximum in $\sigma_{\mathrm{TKE}}$ at small $A_{\mathrm{H}}$ values and the calculations yield a similar feature. This maximum may be related to the onset of bimodal fission, as is discussed in Sec. IV D. TKE distributions for selected mass splits are displayed below in Figs. 8 and 10. 


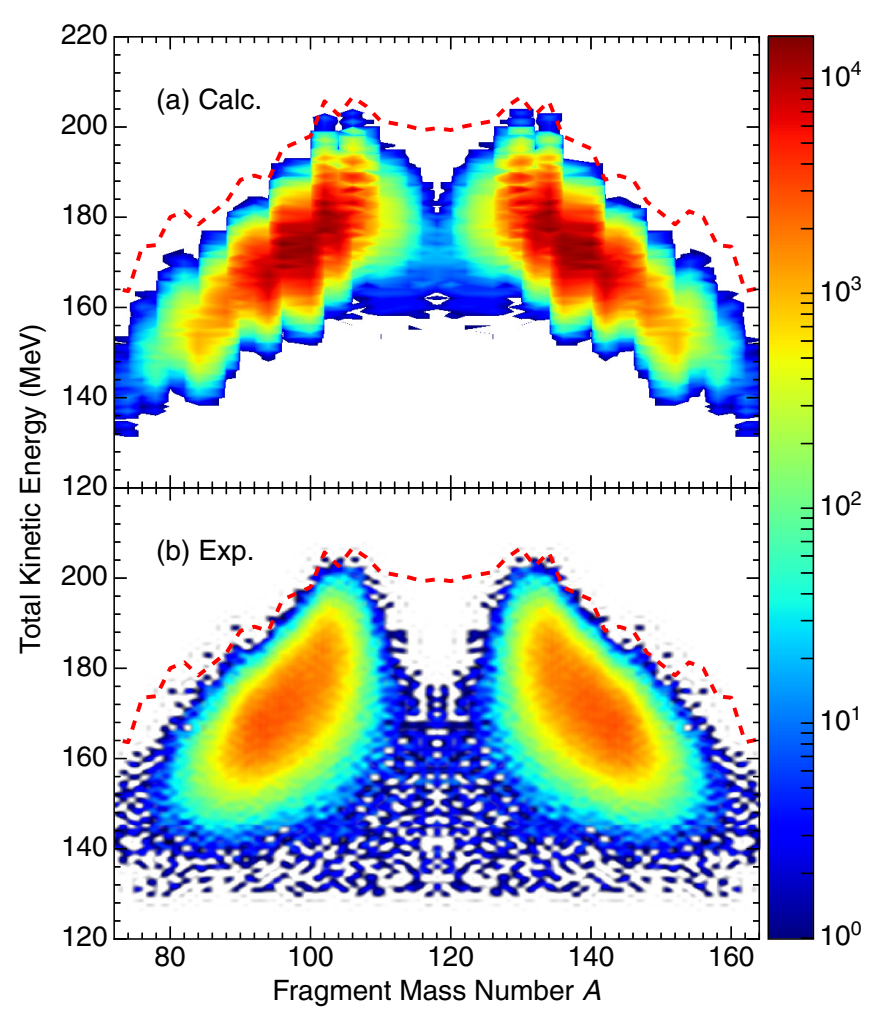

FIG. 2. Number of scission events in log scale vs fissionfragment mass number and total kinetic energy for ${ }^{235} \mathrm{U}\left(n_{\mathrm{th}}, f\right)$. (a) Calculated results with $c_{0}=1.5 \mathrm{fm}$. (b) Experimental data from Ref. [18]. The dashed curve shows $Q$ values for different fragment masses. The experimental number of events is scaled to the same number of events as calculated, namely, $10^{6}$. The jaggedness of the calculated contour plot is due to the selection of even-even mass numbers, and the finiteness of the calculational grid in TKE. The experimental results contain a certain degree of smearing due to uncertainty in measured mass numbers.

Figure 2 shows contour plots of the calculated (a) and measured (b) number of fission events with respect to fragment mass number $A$ and total fragment kinetic energy TKE for ${ }^{235} \mathrm{U}\left(n_{\mathrm{th}}, f\right)$.

The calculation imposes a fixed $N / Z$ ratio (equal to $144 / 92$ for fission of ${ }^{236} \mathrm{U}$ ) for a given fragment mass number $A$. This simplification implies that there is a definite $Q$ value for each mass division, $Q(A)$, which represents the maximum possible TKE value attainable by the calculations. It is shown by the dashed curve in both panels of Fig. 2 and it can be seen that the bulk of the events lie well below this boundary, both theoretically and experimentally. In reality a particular mass division can lead to fragments with a variety of $N / Z$ ratios, leading to a corresponding range of $Q$ values for each $A$.

For a given mass asymmetry (and thus a given $Q$ value), the variation of TKE is counterbalanced by the variation of TXE, the combined excitation of the primary fragments. Consequently, by gating on TKE it is possible to investigate a TXE range of about $10-40 \mathrm{MeV}$, for a given asymmetry. In particular, if, for a given mass partition, the specified TKE value is gradually decreased, starting from the maximally realized value, the available TXE will exhibit a corresponding

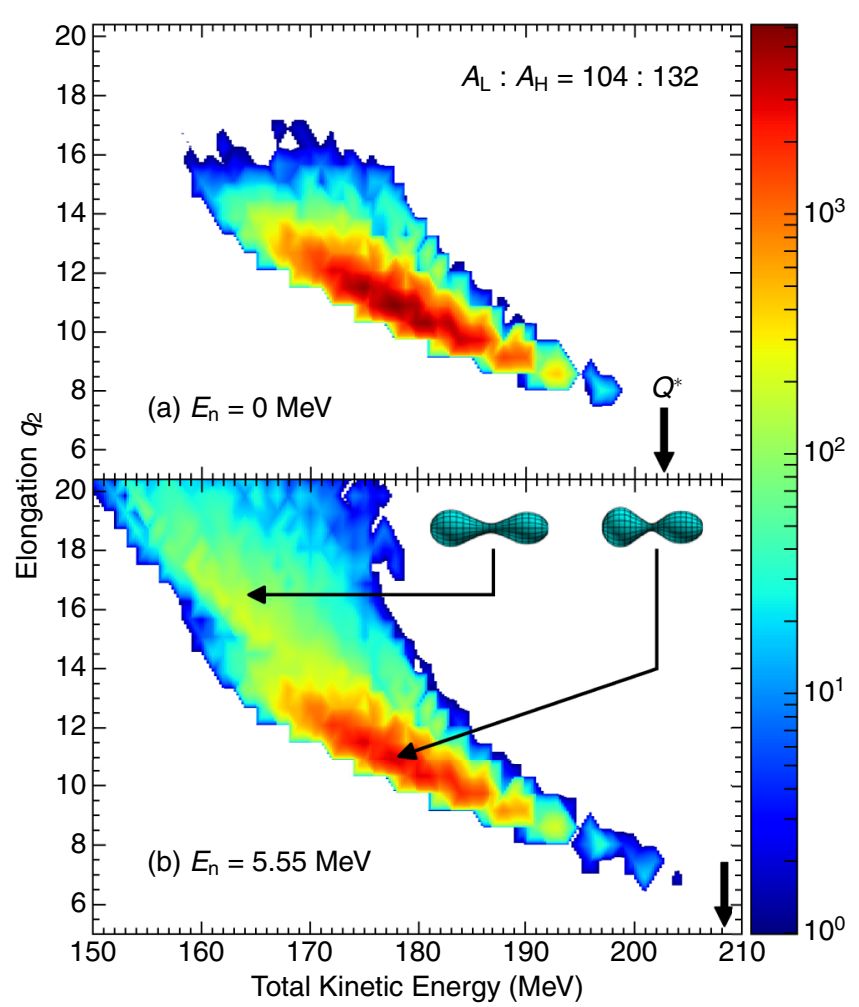

FIG. 3. Number of scission events on a log scale (right bar) for the mass division $A_{\mathrm{L}}: A_{\mathrm{H}}=104: 132$ vs the total kinetic energy TKE and the elongation $q_{2}$, for either thermal fission (a) or $E_{n}=5.55 \mathrm{MeV}$ (b). In (b) typical shapes are shown for the superlong $\left(q_{2} \approx 16.5\right)$ and standard $\left(q_{2} \approx 11\right)$ modes. The vertical arrows indicate the $Q^{*}$ values.

gradual increase. This, in turn, will be reflected in an increase of the mean number of neutrons evaporated from each of the two fragments, $\bar{v}_{\mathrm{L}}$ and $\bar{\nu}_{\mathrm{H}}$, which will provide more detailed experimental information on the origin of $E_{\mathrm{L}}^{*}$ and $E_{\mathrm{H}}^{*}$.

In general, a fission event having a small TKE value (i.e., a large TXE value) is associated with a rather elongated scission configuration, as measured, for example, by the quadrupole moment of the density distribution, $q_{2}$. This feature is illustrated quantitatively in Fig. 3 showing contour plots of the number of fission events versus TKE and $q_{2}$ for the mass split $A_{\mathrm{L}}: A_{\mathrm{H}}=104: 132$ for $E_{n}=0$ (a) and $E_{n}=5.55 \mathrm{MeV}$ (b). At $E_{n}=0$ a wide range of quadrupole moments occur, $8<$ $q_{2}<18$, for the considered mass partition. The most compact scission shapes (having the smallest $q_{2}$ values) are associated with large TKE values close to the $Q$ value, while the most elongated scission shapes (having the largest $q_{2}$ values) have TKE values that are about $40 \mathrm{MeV}$ lower. The scission shapes are thus strongly dependent on the considered TKE value.

In Fig. 3(b) we show that even more elongated scission shapes can occur when more energy is made available by increasing the kinetic energy of the incident neutron. This is related to the appearance of a superlong fission mode. In four-dimensional Langevin calculations [19] the symmetric fission events at low TKE (140-170 MeV) seen in Fig. 2(b), valid for thermal neutrons, could be related to the superlong mode. With increasing excitation energy this fission mode 


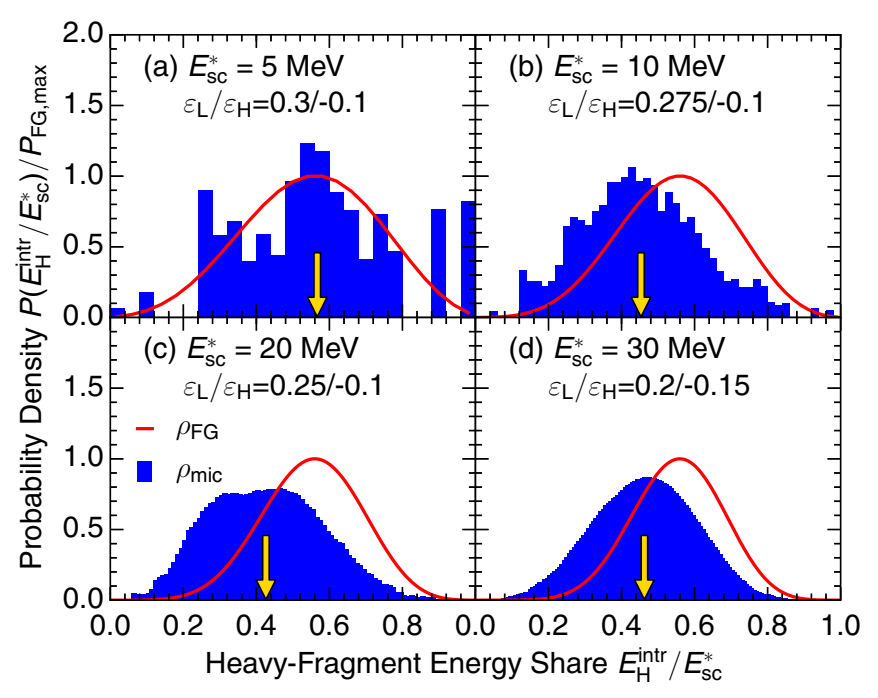

FIG. 4. Probability distribution for the heavy-fragment share of intrinsic energy, $E_{\mathrm{H}}^{\mathrm{intr}} / E_{\mathrm{sc}}^{*}$ [see Eq. (4)], as obtained with microscopic level densities (blue histogram) or Fermi-gas level densities (red curve) for the mass division $A_{\mathrm{L}}: A_{\mathrm{H}}=104: 132$ and a total intrinsic energy $E_{\mathrm{sc}}^{*}=E_{\mathrm{L}}^{\text {intr }}+E_{\mathrm{H}}^{\text {intr }}$ equal to 5 (a), 10 (b), (c) 20 (c), and 30 (d) $\mathrm{MeV}$. The indicated deformations of light and heavy and light, $\varepsilon_{\mathrm{L}} / \varepsilon_{\mathrm{H}}$, are average values at the specified value of $E_{\mathrm{sc}}^{*}$ and the arrows mark the average energy share obtained with microscopic level densities.

becomes important also for asymmetric fission, as is discussed in Sec. IV D.

\section{Intrinsic excitation energies}

In the present calculations, the intrinsic excitation energy available at scission, $E_{\mathrm{sc}}^{*}$, is divided statistically between the two proto-fragments, as proposed in Ref. [7]. Thus the probability distribution for the intrinsic excitation of the heavy fragment, $E_{\mathrm{H}}^{\mathrm{intr}}$, has a microcanonical form:

$$
P\left(E_{\mathrm{H}}^{\mathrm{intr}} ; E_{\mathrm{sc}}^{*}\right) \sim \rho_{\mathrm{H}}\left(E_{\mathrm{H}}^{\mathrm{intr}} ; \varepsilon_{\mathrm{H}, \mathrm{sc}}\right) \rho_{\mathrm{L}}\left(E_{\mathrm{sc}}^{*}-E_{\mathrm{H}}^{\mathrm{intr}} ; \varepsilon_{\mathrm{L}, \mathrm{sc}}\right),
$$

where $\rho_{\mathrm{H}}\left(E^{*}\right)$ and $\rho_{\mathrm{L}}\left(E^{*}\right)$ are the effective level densities of the heavy and light proto-fragments evaluated at their respective shapes at scission. Figures 4 and 5 show the energy distribution function $P\left(E_{\mathrm{H}}^{\mathrm{intr}} ; E_{\mathrm{sc}}^{*}\right)$ for typical scission shapes for fission of ${ }^{236} \mathrm{U}$ into ${ }^{104} \mathrm{Zr}+{ }^{132} \mathrm{Te}$ and ${ }^{88} \mathrm{Se}+{ }^{148} \mathrm{Ce}$, respectively, in each case for four different values of the total available energy, $E_{\mathrm{sc}}^{*}=5,10,20,30 \mathrm{MeV}$.

In Figs. 4 and 5, the distribution functions in Eq. (4), based on microscopic level densities, are compared to the corresponding distribution functions based on simple Fermigas level densities. Both types of level density yield rather broad distributions due to the smallness of the nuclear system. However, while the Fermi-gas form gives smooth distributions that peak where the energy share equals the mass share, $E_{\mathrm{H}}^{\mathrm{intr}} / E_{\mathrm{sc}}^{*}=A_{\mathrm{H}} / A_{0}$, the microscopic level densities lead to distributions that exhibit significant irregularities at low total excitation energies. Furthermore, importantly, at low values of $E_{\mathrm{sc}}^{*}$ the most probable fragment excitation may differ significantly from the macroscopic expectation given by the

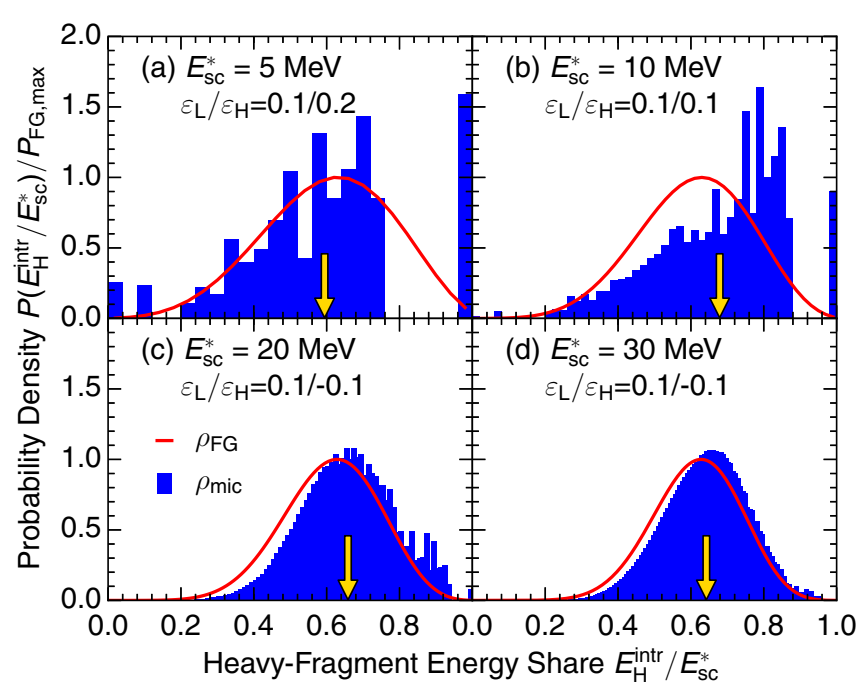

FIG. 5. Similar to Fig. 4 but for $A_{\mathrm{L}}: A_{\mathrm{H}}=88: 148$.

Fermi-gas form. Generally, as the total excitation energy is increased, these nuclear-structure effects diminish and the microscopic energy distribution grows ever smoother as it gradually approaches the Fermi-gas result.

In the example shown in Fig. 4, the heavy fragment, ${ }^{132} \mathrm{Te}$, is close to being doubly magic and has a large negative shell energy. It therefore requires a relatively high excitation energy to approach the Fermi-gas result. Furthermore, the low level density of this fragment causes the light fragment, ${ }^{104} \mathrm{Zr}$, to be favored in the energy sharing at most excitation energies. For example, when the available total excitation energy is $E_{\mathrm{sc}}^{*}=10 \mathrm{MeV}$ [Fig. 4(b)], on the average about $6 \mathrm{MeV}$ goes to the light fragment ${ }^{104} \mathrm{Zr}$ and only about $4 \mathrm{MeV}$ goes to the heavy fragment ${ }^{132} \mathrm{Te}$, while the Fermi-gas level densities lead to the reverse energy partitioning.

The other example (Fig. 5) is a somewhat more asymmetric mass division, ${ }^{88} \mathrm{Se}+{ }^{148} \mathrm{Ce}$, and some favoring of the heavy fragment is apparent, in particular at low values of $E_{\mathrm{sc}}^{*}$. A quite spectacular situation emerges at the lowest energy shown, $E_{\mathrm{sc}}^{*}=5 \mathrm{MeV}$, where it is predicted that the heavy fragment acquires all the energy with a non-negligible probability $(\approx 14 \%)$. This is partly due to large pairing gaps (thus low level density) for the light fragment ${ }^{88} \mathrm{Se}$, causing the heavy fragment ${ }^{148} \mathrm{Ce}$ to be favored in the energy partitioning.

The excitation energy partition between the heavy and the light fragments may thus change significantly with the amount of total excitation energy available and, furthermore, the partition scenario may change dramatically from one pair of fission fragments to another.

With this background, we now consider the average values of the intrinsic excitation energies as well as the distortion energies of the proto-fragments resulting from the ensemble of scission configurations obtained with the METROPOLIS shape evolution. Figure 6 shows the average intrinsic energy of the heavy and light fragment versus the specified value of TKE, for eight selected fragment-pair combinations. These examples are chosen from the full range of mass divisions: 


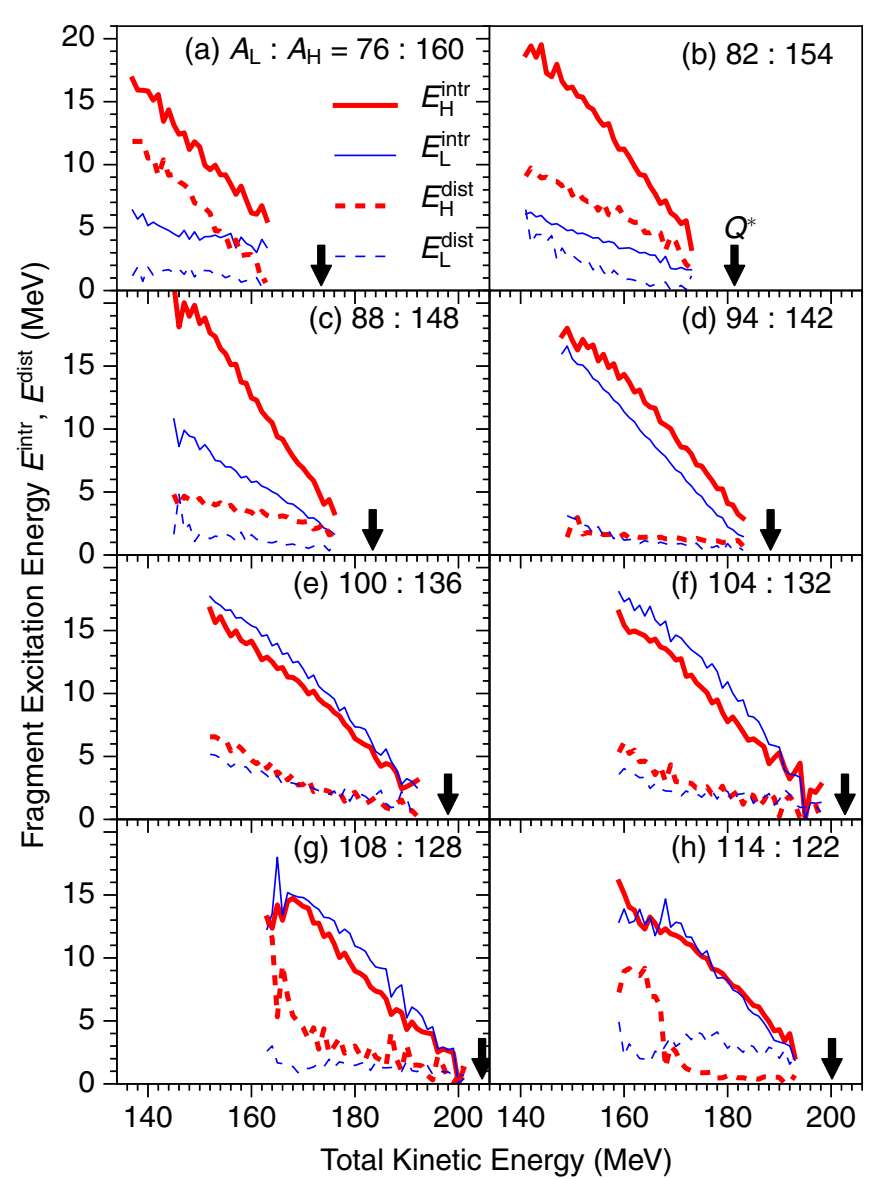

FIG. 6. The average of the light and heavy-fragment intrinsic energies, $E_{\mathrm{L}}^{\text {intr }}$ (solid blue) and $E_{\mathrm{H}}^{\text {intr }}$ (solid red), and the average fragment distortion energies, $E_{\mathrm{L}}^{\text {dist }}$ (dashed blue) and $E_{\mathrm{H}}^{\text {dist }}$ (dashed red), as functions of the total kinetic energy TKE for eight mass divisions $A_{\mathrm{L}}: A_{\mathrm{H}}=76: 160$ (a), 82:154 (b), 88:148 (c), 94:142 (d), 100:136 (e), 104:132 (f), 108:128 (g), and 114:122 (h), for ${ }^{235} \mathrm{U}\left(n_{\mathrm{th}}, f\right)$. The arrows point to the $Q^{*}$ values.

$A_{\mathrm{L}}: A_{\mathrm{H}}=76: 160$ (a), 82:154 (b), 88:148 (c), 94:142 (d), 100:136 (e), 104:132 (f), 108:128 (g), and 114:122 (h).

Large nuclear-structure effects are apparent. In particular, the large share of intrinsic excitation given to the heavy fragment in panel (c) $(88: 148)$ can be understood from Fig. 5, and the fact that the light fragment receives the largest energy share in panel (f) (104:132) can be understood from Fig. 4, as discussed above.

\section{Distortion energy}

As discussed above, the distribution of intrinsic energy in a proto-fragment, $E_{i}^{\text {intr }}$, varies with the specified value of TKE. The fragment distortion energies, $E_{i}^{\text {dist }}$, are also sensitive to the specified TKE because the sum of the total collective kinetic energy and the total distortion energy must equal the total available energy minus the total intrinsic energy:

$$
\mathrm{TKE}+E_{\mathrm{L}}^{\mathrm{dist}}+E_{\mathrm{H}}^{\mathrm{dist}}=Q_{\mathrm{LH}}^{*}-E_{\mathrm{sc}}^{*} .
$$

Consequently, at the highest values of TKE the energy balance does not leave much room for fragment distortion.

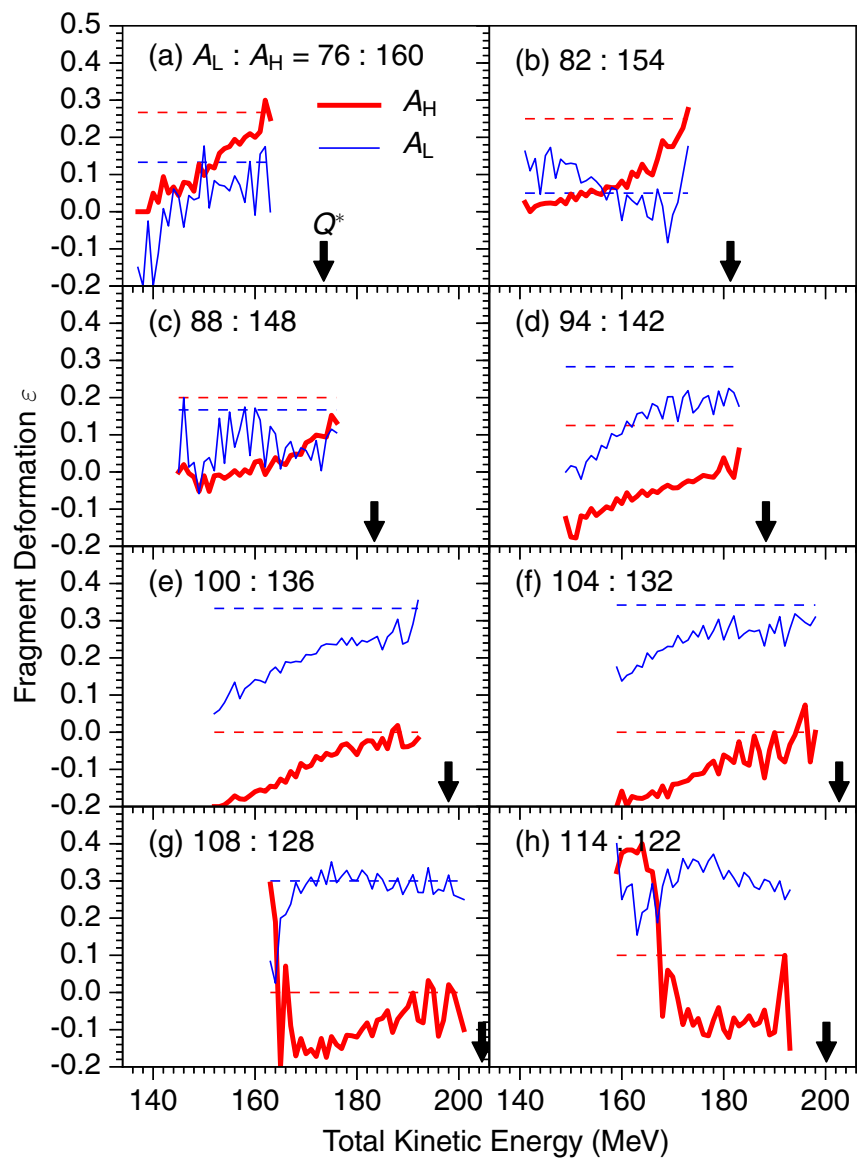

FIG. 7. Average light and heavy-fragment deformations at scission, $\varepsilon_{\mathrm{L}}$ (thin blue curves) and $\varepsilon_{\mathrm{H}}$ (thick red curves), vs the total kinetic energy TKE for the same fragment combinations shown in Fig. 6. The dashed horizontal lines show the corresponding equilibrium deformations. The arrows point to the $Q^{*}$ values. The jaggedness of the curves is caused by the sampling on a discrete lattice of $3 \mathrm{QS}$ shapes. For $A_{\mathrm{L}}=114$ the ground-state minimum at $\varepsilon=-0.25$ (outside the scale) coexists with an isomeric minimum at $\varepsilon=0.23$.

Conversely, the lowest TKE values are associated with large elongations of the scission configurations and significant distortions of the proto-fragments. The relationship between TKE and the fragment distortion energies is illustrated in Fig. 6 for eight different mass partitions, and the TKE dependence of the proto-fragment shapes is illustrated in Fig. 7 for the same cases.

The distortion energy resulting from a certain deformation change depends strongly on the structure of the specific fragment considered. For example, for the fragment ${ }^{160} \mathrm{Sm}$ a deformation change from the ground-state value $\varepsilon=0.25$ to 0.0 [Fig. 7(a)] yields a distortion energy of $E^{\text {dist }}=11 \mathrm{MeV}$ [Fig. 6(a)], while the same deformation change for ${ }^{94} \mathrm{Kr}$ [Fig. 7(d)] yields a much smaller distortion energy, $E^{\text {dist }}=$ $3 \mathrm{MeV}$ [Fig. 6(d)]. This is because the deformation energy curve $U(\varepsilon)$ for ${ }^{160} \mathrm{Sm}$ has a robust (i.e., stiff) prolate minimum, while that for ${ }^{94} \mathrm{Kr}$ is rather soft around its prolate minimum (with respect to both $\varepsilon$ and $\gamma$ ). 
For most mass divisions, the distortion energy of the heavy fragment is consistently larger than that of the light fragment, $E_{\mathrm{H}}^{\text {dist }}>E_{\mathrm{L}}^{\text {dist }}$. This difference grows with increasing mass asymmetry and the largest difference is found for $A_{\mathrm{L}}: A_{\mathrm{H}}=76: 160$, while there is almost no difference for $A_{\mathrm{L}}: A_{\mathrm{H}}=94: 142$, 100:136, or 104:132. An exception where the light fragment obtains a larger distortion energy is for $A_{\mathrm{L}}: A_{\mathrm{H}}=114: 122$ at high TKE. In this case the light fragment, ${ }^{114} \mathrm{Ru}$, has two shape-coexisting minima, one prolate $(\varepsilon \approx$ 0.23 ) and one oblate $(\varepsilon \approx-0.25)$, where the excited prolate shape is more frequently populated in the METROPOLIS walk than the oblate ground-state minimum. This gives the light fragment a substantial distortion energy also at high TKE.

We also note that the distortion energy is systematically smaller than the intrinsic energy for given fragment mass partition and TKE, $E_{i}^{\text {dist }}<E_{i}^{\text {intr }}$, but the relative contribution from the distortion energy to the total excitation energy of a fragment varies substantially from one fragment to another. For example, for $A_{\mathrm{L}}: A_{\mathrm{H}}=94: 142$ both fragments receive only 10-15\% from the distortion energy, while for 82:154 the light fragment receives almost half of its final excitation energy from the distortion energy at the lowest TKE values (but the contribution drops to only about $10 \%$ at the highest TKE values).

For the two most symmetric cases studied, $A_{\mathrm{L}}: A_{\mathrm{H}}=108: 128$ and 114:122, we note that the average deformation of the heavy fragment increases drastically at lowest values of TKE. This is due to the presence of the superlong fission mode which is primarily affecting the symmetric region, but plays a role also for asymmetric fission at sufficiently high excitation energies, as is discussed in Sec. IV D.

In general, our results reveal a quite complex, structuredependent variation of both the intrinsic energy and the distortion energy with the fragment identity as well as with TKE.

\section{NEUTRON MULTIPLICITIES}

After the primary fission fragments have been fully accelerated by their mutual Coulomb repulsion and their shapes have relaxed to their equilibration form, they typically deexcite by (typically sequential) neutron evaporation followed by photon radiation. The mean number of neutrons emitted from a particular fragment species, $\bar{v}_{i}$, presents a convenient (and observable) measure of the degree of its initial excitation, $E_{i}^{*}=E_{i}^{\mathrm{intr}}+E_{i}^{\text {dist }}$.

Therefore, in the present paper, we calculate neutron evaporation from the excited fragments. We employ the method described in our earlier study [7] (which was adapted from the treatment in Ref. [20]) using the effective microscopic level densities of the daughter nuclei. Thus we assume that the kinetic-energy spectrum of an evaporated neutron is given by

$$
d N_{n}\left(\epsilon_{n}\right) / d \epsilon_{n} \sim \rho^{\prime}\left(E_{\max }^{\prime}-\epsilon_{n}\right) \epsilon_{n},
$$

where $E_{\max }^{\prime}=E^{*}-S_{n}^{\prime}$ is the maximum excitation in the daughter nucleus (corresponding to the evaporation of a neutron having vanishing kinetic energy $\left.\epsilon_{n}\right)$ and $\rho^{\prime}\left(E^{\prime}\right)$ is its level density.

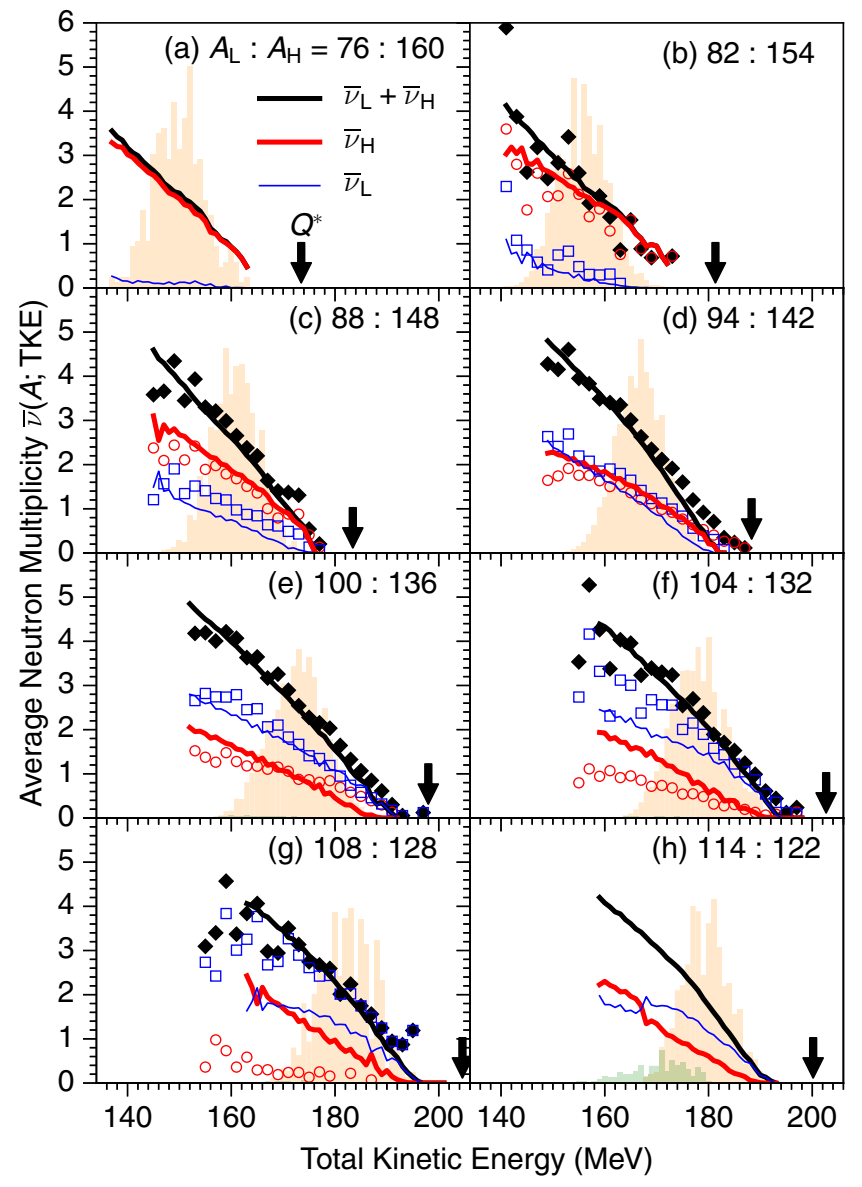

FIG. 8. Average multiplicity of neutrons evaporated from the light or heavy fragment in ${ }^{235} \mathrm{U}\left(n_{\mathrm{th}}, f\right)$ as a function of TKE, $\bar{v}_{\mathrm{L}}(A, \mathrm{TKE})$, and $\bar{\nu}_{\mathrm{H}}(A, \mathrm{TKE})$, for the eight mass divisions shown in Fig. 6. Calculated: $\bar{v}_{\mathrm{L}}$ (thin blue lines), $\bar{v}_{\mathrm{H}}$ (thick red lines), $\bar{\nu}_{\mathrm{L}}+\bar{v}_{\mathrm{H}}$ (black lines). Measured [8]: $\bar{\nu}_{\mathrm{L}}$ (open blue squares), $\bar{\nu}_{\mathrm{H}}$ (open red circles), $\bar{v}_{\mathrm{L}}+\bar{v}_{\mathrm{H}}$ (black squares). The histograms show the calculated TKE distributions, $P(\mathrm{TKE}$ ), for the standard (orange) and the superlong (green) fission modes. The arrows point to the $Q^{*}$ values.

For thermal fission, $E_{n} \approx 0$, the average neutron multiplicity from each fragment species is calculated for specified values of TKE (Sec. IV A) and for specified values of TXE (Sec. IV B). Corresponding results for $E_{n}=5.55 \mathrm{MeV}$ are presented subsequently (Sec. IV C). In Sec. IV D we discuss how the superlong fission mode plays an ever more important role with increasing excitation energy.

\section{A. TKE-gated neutron multiplicities}

Figure 8 shows the calculated average neutron multiplicity from the light and the heavy fragments for specified TKE, $\bar{v}\left(A_{\mathrm{L}} ; \mathrm{TKE}\right)$ and $\bar{v}\left(A_{\mathrm{H}} ; \mathrm{TKE}\right)$, as well as their sum, for the same eight mass divisions considered in Figs. 6 and 7. Also shown (where available) are the experimental results reported by Göök et al. [8]. The calculated TKE distribution is shown for each mass split by the shaded area. In Sec. III B (Fig. 1) we discussed the averages and widths of this distribution. 
We note that only relatively few events contribute in the tails of the TKE distribution, i.e., for small or large TKE values.

As brought out in Fig. 8, the calculated multiplicities, $\bar{\nu}_{\mathrm{L}}(\mathrm{TKE})$ and $\bar{\nu}_{\mathrm{H}}(\mathrm{TKE})$, agree well with the measured values for asymmetric divisions, $A_{\mathrm{H}} \geqslant 136$. For the more symmetric mass splits the calculations substantially overestimate the number of neutrons emitted from the heavy fragment, most severely for the mass split 108:128. This interval in fragment mass number of discrepancy in neutron multiplicity coincides with that of the discrepancy found for the average TKE.

For very asymmetric divisions the heavy fragment receives most of the excitation energy (see Fig. 6) and, as a result, it contributes almost all of the neutrons. This feature is most pronounced for the most asymmetric case displayed, $A_{\mathrm{L}}: A_{\mathrm{H}}=76: 160$, but it is also clearly present for $82: 154$ and, to a smaller degree, for $88: 148$. For $94: 142$ the two mean multiplicities are very similar, even though the heavy fragment is $\approx 50 \%$ larger than the light one. Closer to symmetry (the four lowest panels of Fig. 8), where the microscopic effects tend to favor the light fragment in the energy division, the neutron multiplicity is dominated by the light fragment. This gradual change in the mean neutron multiplicity as a function of the mass asymmetry is also present in the TKE-averaged results, as was previously discussed [7].

The calculated dependence of the total excitation energy of a given fragment, $E_{i}^{*}=E_{i}^{\text {intr }}+E_{i}^{\text {dist }}$, on the specified value of TKE (Fig. 6), provides an understanding of how the mean neutron multiplicity varies with TKE for the various fragment masses.

For the most asymmetric mass division considered, $A_{\mathrm{L}}: A_{\mathrm{H}}=76: 160$ [Fig. 8(a)], 70-80\% of the total excitation energy is carried by the heavy fragment. Because the light fragment is then typically insufficiently excited for neutron evaporation to occur, practically all of the neutrons originate from the heavy fragment, for all values of TKE.

For several mass divisions the dependence of $\bar{v}$ on TKE is almost linear. An interesting exception is the threshold effect found for the light fragment in the 82:154 division [Fig. 8(b)], an effect also seen in the measurements [8]. For large values of TKE all neutrons are emitted from the heavy fragment, and evaporation from the light fragment sets in smoothly as TKE is lowered, resulting in a gradual increase of $\bar{\nu}_{\mathrm{L}}$ from zero to about one as TKE is decreased from about 160 to $145 \mathrm{MeV}$.

For the 88:148 mass division the light fragment is calculated to emit on the average somewhat more than one neutron at the smallest TKE values, while the heavy fragment dominates, emitting up to about three neutrons at small TKE.

For $A_{\mathrm{L}}: A_{\mathrm{H}}=94: 142$ one third of the total excitation energy is concentrated in the light fragment at high TKE, and its share increases smoothly with decreasing TKE towards an equal share for both fragments at the smallest TKE. The effect on the neutron multiplicity is seen in Fig. 8(d): At high TKE the neutron evaporation is coming only from the heavy fragment, but as TKE is decreased the ratio changes smoothly, leading towards equal contributions from the light and heavy fragments at small TKE. To some degree, this is also seen in the measured neutron multiplicities. However, at the highest
TKE values, close to $Q^{*}$, neutron multiplicities up to 0.2 have been measured, while the calculated values are strictly zero for energies in the range from $Q^{*}$ to $Q^{*}-S_{n}$. Such a difference may be due to the measured mass numbers having a finite resolution (full width at half maximum $\approx 5$ [8]), leading to a corresponding dispersion in the $Q^{*}$ value.

Most neutrons are emitted from the light fragment for the two divisions 100:136 and 104:132. For these cases we have $\bar{\nu}_{\mathrm{L}}>\bar{v}_{\mathrm{H}}$ for all TKE values, with the difference decreasing with decreasing TKE for 104:132. The shrinking difference between $\bar{v}_{\mathrm{L}}$ and $\bar{v}_{\mathrm{H}}$ with decreasing TKE develops into a crossing of the calculated values of $\bar{\nu}_{\mathrm{L}}$ and $\bar{\nu}_{\mathrm{H}}$ for the two most symmetric cases studied, 108:128 and 114:122. With higher incoming neutron energy (see the results displayed in Fig. 10) the crossing becomes more pronounced and the mechanism for the crossing will be discussed in Sec. IV C.

The small number of neutrons evaporated from fragments with mass numbers $A \approx 132$ is usually attributed to the double-shell closure at $N=82$ and $Z=50$, where the $Q$ value has a maximum. The present model restricts the $N: Z$ ratio in the fragments to be that of the fissioning system. As a consequence, for fragments having mass number 132, the species closest to the doubly magic nucleus ${ }^{132} \mathrm{Sn}$ is ${ }^{132} \mathrm{Te}$ having $N=80$ and $Z=52$, which is two neutrons and two protons away from magicity. A generalized model that allows the $N: Z$ ratio to take on different values in the two fragments (see, e.g., [21]) would quite likely lead to a preferential production of ${ }^{132} \mathrm{Sn}$ relative to ${ }^{132} \mathrm{Te}$ for $A_{\mathrm{H}}=132$. Such nuclei, having two closed spherical shells, are more resistant to deformations than nuclei situated four nucleons away and would therefore have a lower distortion energy than that obtained in the present calculation. Furthermore, the spherical magicity would imply a smaller level density, causing the heavy fragment to receive a smaller share of the statistical excitation energy. Consequently, the present calculations can be expected to somewhat overestimate the total excitation of fragments with $A_{\mathrm{H}} \approx 132$ and, therefore, to also overestimate the resulting neutron multiplicity.

The deviation between calculations and measurement for the split $A_{\mathrm{L}}: A_{\mathrm{H}}=108: 128$ is more difficult to understand. For example, at $\mathrm{TKE} \approx 165 \mathrm{MeV}$ (corresponding to a total excitation energy of TXE $\approx 40 \mathrm{MeV}$ ) the calculations yield $\bar{v}_{\mathrm{H}} \approx 2.0$, whereas the reported value [8] is only 0.3. Although this mass split is in the symmetric region where TKE is systematically overestimated, the deviation between measurement and calculation seems substantial.

It is interesting to compare these data to the neutron multiplicity data for incoming neutron energy $E_{n}=5.55 \mathrm{MeV}$. For mass division 108:128, the increase of $E_{n}$ from 0.5 to $5.55 \mathrm{MeV}$ results in a decrease in TKE of about $4 \mathrm{MeV}$ (due to more elongated scission shapes) which, together with the higher neutron energy, leads to an increase of TXE by about $9 \mathrm{MeV}$. This in turn results in about 0.9 additional neutrons evaporated from the heavy fragment (using smoothed values read off from Figs. 8 and 11 in Ref. [14]). This can be compared to the correlation data displayed in Fig. 8(g) where an increase in TXE of $9 \mathrm{MeV}$ (a decrease in TKE, e.g., from 180 to $171 \mathrm{MeV}$ ) is seen to result in only about 0.2 additional neutrons emitted from the heavy fragment. Our calculations 


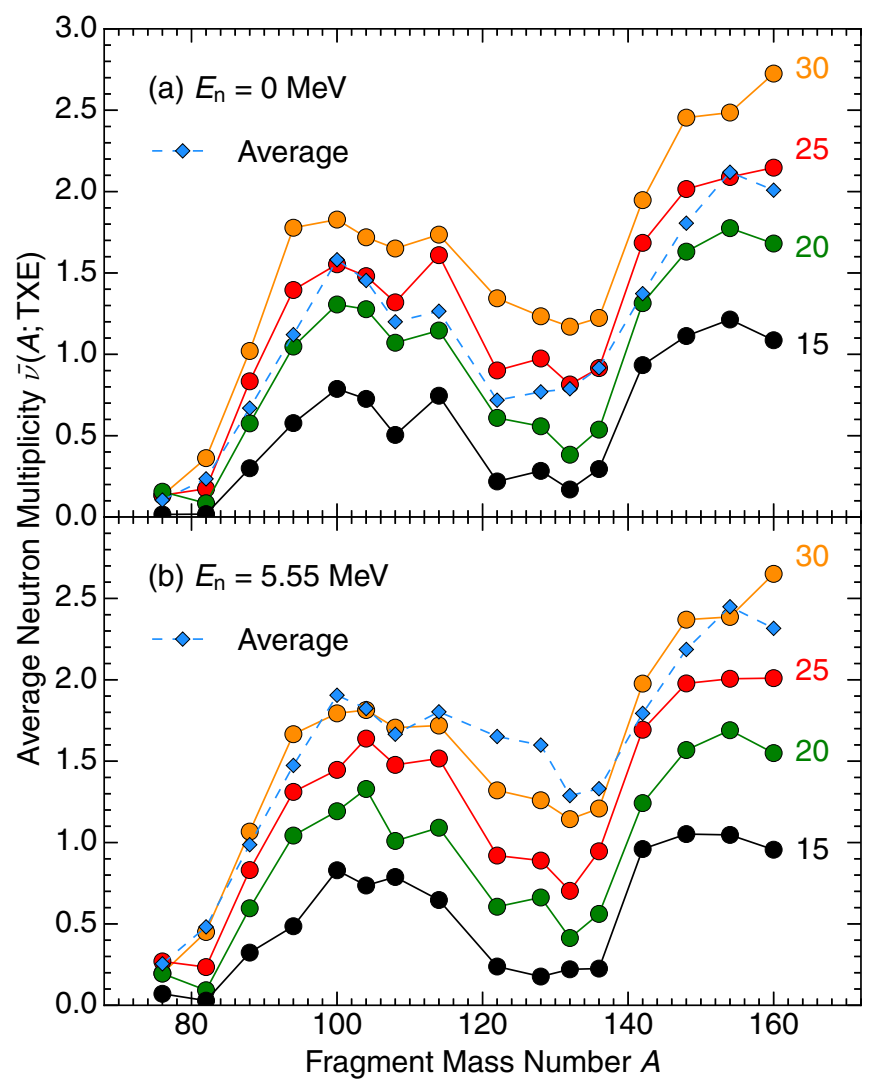

FIG. 9. Calculated average neutron multiplicity for ${ }^{235} \mathrm{U}\left(n_{\text {th }}, f\right)$ for four specified values of TXE (indicated in MeV), as a function of the fission-fragment mass number, $\bar{v}(A, T X E)$, for either thermal fission (a) or $E_{n}=5.5 \mathrm{MeV}$ (b). The TXE-averaged neutron multiplicity $\bar{v}(A)$ [see Eq. (7)] is also shown in each panel (blue diamonds connected by dashed lines).

yield about equal average number of neutrons emitted for the two ways of increasing TXE by $9 \mathrm{MeV}$, namely, about 0.8 extra neutrons.

\section{B. TXE-gated neutron multiplicities}

When the total excitation energy TXE is fixed, then all different fragment pairs have the same amount of excitation energy to share (namely, TXE), which would make it possible to determine the variation of $\bar{v}(A$; TXE) as TXE is changed and study how the sawtooth feature evolves with excitation energy.

It is elementary to obtain $\bar{v}(A$; TXE) from $\bar{v}(A$; TKE) because TXE $=Q_{\mathrm{LH}}^{*}-$ TKE for a given light-heavy mass split, so $\bar{v}(A ; \mathrm{TXE})=\bar{v}\left(A ; \mathrm{TKE}=Q_{\mathrm{LH}}^{*}-\mathrm{TXE}\right)$ where the fragment mass number $A$ determines the $\mathrm{L}-\mathrm{H}$ division.

Figure 9 shows the calculated $\bar{v}(A$; TXE) for four different values of the total excitation energy, TXE $=15,20,25$, $30 \mathrm{MeV}$, for either $E_{n}=0$ (a) or $E_{n}=5.55 \mathrm{MeV}$. The result is very similar in the two cases because it is the specified TXE value (rather than $E_{n}$ ) that determines the properties of the fragments.

As discussed in detail above, the specified total excitation energy TXE is being divided quite unevenly between the two fragments due to the complexity of the separate contributions from intrinsic energy and distortion energy. The characteristic sawtooth behavior appears for all four TXE values.

With increasing TXE the excitation energy of each fragment increases, resulting in larger neutron multiplicities from both fragments. However, the increase of the excitation energy of a fragment is not linear in TXE, as was discussed in Secs. IIIC and III D. For example, for $A_{\mathrm{L}}: A_{\mathrm{H}}=104: 132$ an increase of TXE by $5 \mathrm{MeV}$ from 15 to $20 \mathrm{MeV}$ results in an energy increase of about $3.5 \mathrm{MeV}$ in the light fragment and $1.5 \mathrm{MeV}$ in the heavy fragment, leading to multiplicity increases of about $\Delta \bar{\nu}_{\mathrm{L}}=0.6$ and $\Delta \bar{\nu}_{\mathrm{H}}=0.2$. By contrast, a similar increase in TXE from 25 to $30 \mathrm{MeV}$ causes an energy increase of about $2.5 \mathrm{MeV}$ in both fragments leading to $\Delta \bar{\nu}_{\mathrm{L}}=\Delta \bar{\nu}_{\mathrm{H}}=0.3$. This evolution away from light-fragment dominance with increasing TXE is caused primarily by the specific energy dependence of the level densities in the distorted proto-fragments which reduces the favoring of the light fragment in the sharing of the intrinsic energy at scission, as is seen in Figs. 4(b) and 4(c).

The neutron multiplicity from more asymmetric divisions shows a less dramatic evolution with TXE. For example, for $A_{\mathrm{L}}: A_{\mathrm{H}}=82: 154$ the multiplicity increases are $\Delta \bar{\nu}_{\mathrm{L}}=0.1$ and $\Delta \bar{\nu}_{\mathrm{H}}=0.6$ when TXE is increased from 5 to $10 \mathrm{MeV}$, and $\Delta \bar{v}_{\mathrm{L}}=0.2$ and $\Delta \bar{\nu}_{\mathrm{H}}=0.4$ when TXE is increased from 25 to $30 \mathrm{MeV}$.

When TXE is increased from 15 to $30 \mathrm{MeV}$ for $A_{\mathrm{L}}: A_{\mathrm{H}}=76: 160$ almost all increase in excitation energy goes to the heavy fragment. This results in the large increase in heavy-fragment neutron multiplicity, $\Delta \bar{\nu}_{\mathrm{H}}=1.7$, as compared to only $\Delta \bar{\nu}_{\mathrm{L}}=0.3$ for the light fragment.

Figure 8 also shows the unconstrained neutron multiplicity, $\bar{v}(A)$, for both thermal fission, $E_{n} \approx 0$ (a), and the higher energy considered in Sec. IV C, $E_{n}=5.55 \mathrm{MeV}$ (b). The unconstrained multiplicity can be regarded as a weighted average of the TXE-constrained multiplicity:

$$
\bar{v}(A)=\frac{\int N(A ; \mathrm{TXE}) \bar{v}(A ; \mathrm{TXE}) d \mathrm{TXE}}{\int N(A ; \mathrm{TXE}) d \mathrm{TXE}},
$$

where $N(A$; TXE) denotes the number of events leading to the specified value of TXE. For thermal fission the average TXE value is found to be around $25 \mathrm{MeV}$ for most the $A$ values shown, except for $A_{\mathrm{L}}: A_{\mathrm{H}}=88: 148$ and 94:142 where $\overline{\mathrm{TXE}}=21-22 \mathrm{MeV}$. We note that $\bar{v}(A)$ agrees very well with $\bar{v}(A ; \overline{\mathrm{TXE}})$ for both thermal fission and for $E_{n}=5.55 \mathrm{MeV}$ for which we have $\overline{\mathrm{TXE}} \approx 30 \mathrm{MeV}$.

\section{Fission at higher energy}

We now discuss in more detail our results for the higher incoming energy, $E_{n}=5.55 \mathrm{MeV}$, which is close to the threshold for second-chance fission. This neutron energy has been studied experimentally [14].

The calculated neutron multiplicity, averaged over all fission mass divisions and TKE values, increases from $\bar{v}=2.41$ for thermal fission to $\bar{v}=3.20$ for $E_{n}=5.55 \mathrm{MeV}$. This is in very good agreement with corresponding measured values of $\bar{v}=2.42$ and 3.12, respectively [22]. 


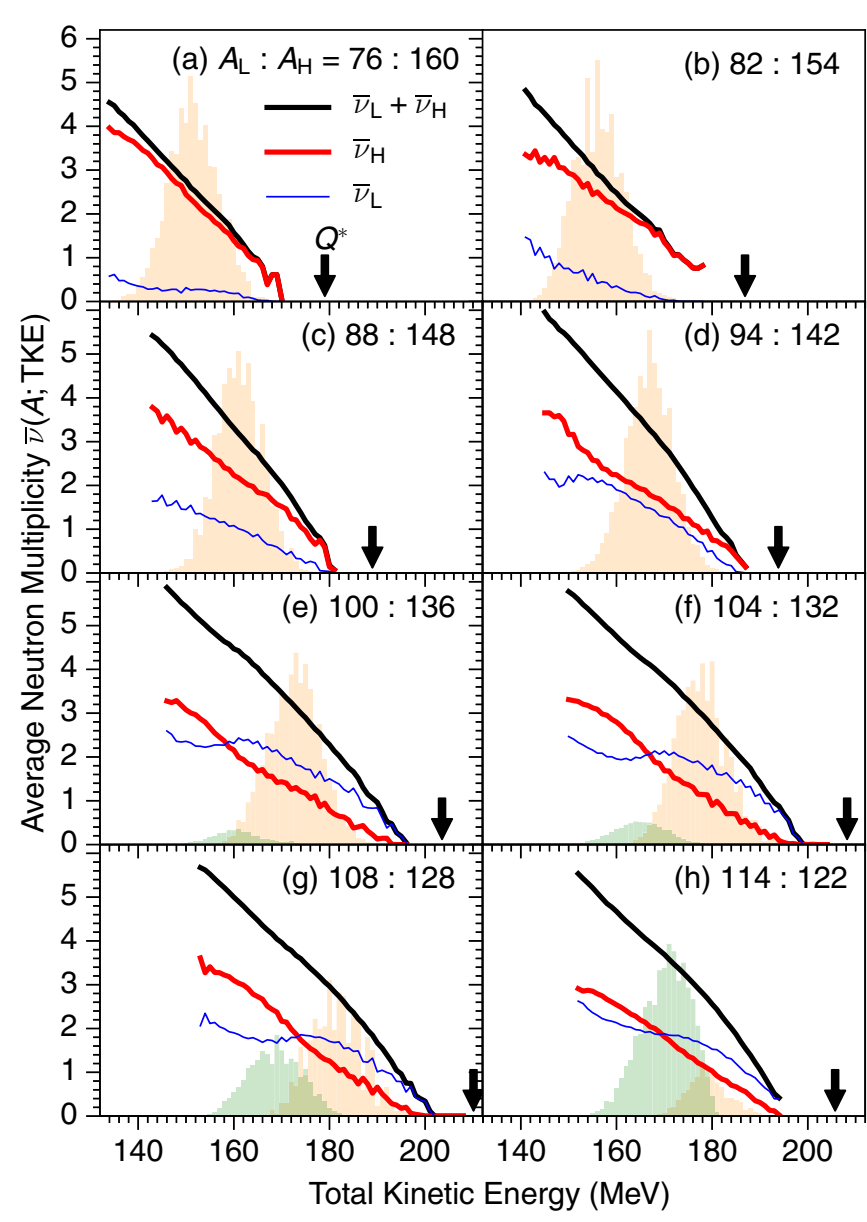

FIG. 10. Same as Fig. 8 but for $E_{n}=5.55 \mathrm{MeV}$.

Figure 10 shows the TKE-gated mean neutron multiplicity, $\bar{v}(A$; TKE), for the same eight mass divisions as studied above. When the energy of the incoming neutron increases, the $Q$ value increases correspondingly because excitation energy of the fissioning nucleus is $E_{0}^{*}=S_{n}+E_{n}$. Thus TKE+TXE is increased by $E_{n}$ and we find that the average TKE changes very little and most of the additional energy goes to TXE. As a consequence, the shape evolution is able to explore a wider domain of the potential-energy landscape and the system gains access to valleys that lead to more elongated scission shapes. This feature will be reflected in a bimodal character of the TKE distribution (see Sec. IV D).

For the three most asymmetric divisions, Figs. 10(a)-10(c), the behavior of $\bar{v}(A$; TKE) is similar to the thermal result for both $\bar{\nu}_{\mathrm{L}}$ and $\bar{\nu}_{\mathrm{H}}$ [see Figs. 8(a)-8(c)], except for an overall increase due to the increased excitation of the primary fission fragment. On average, the neutron multiplicity increases by $0.3-0.4$ for both the light and the heavy fragments.

This smooth evolution with $E_{n}$ may be contrasted with the behavior for the less asymmetric divisions [Figs. 10(d)-10(h)] where qualitative changes are apparent. For the four least asymmetric cases, it is especially noticeable that $\bar{v}\left(A_{\mathrm{L}} ; \mathrm{TKE}\right)$ and $\bar{v}\left(A_{\mathrm{H}}\right.$; TKE) cross so the heavy fragment becomes dominant at low TKE, and the light fragment emits most neutrons at higher TKE. Similar crossings were present already for

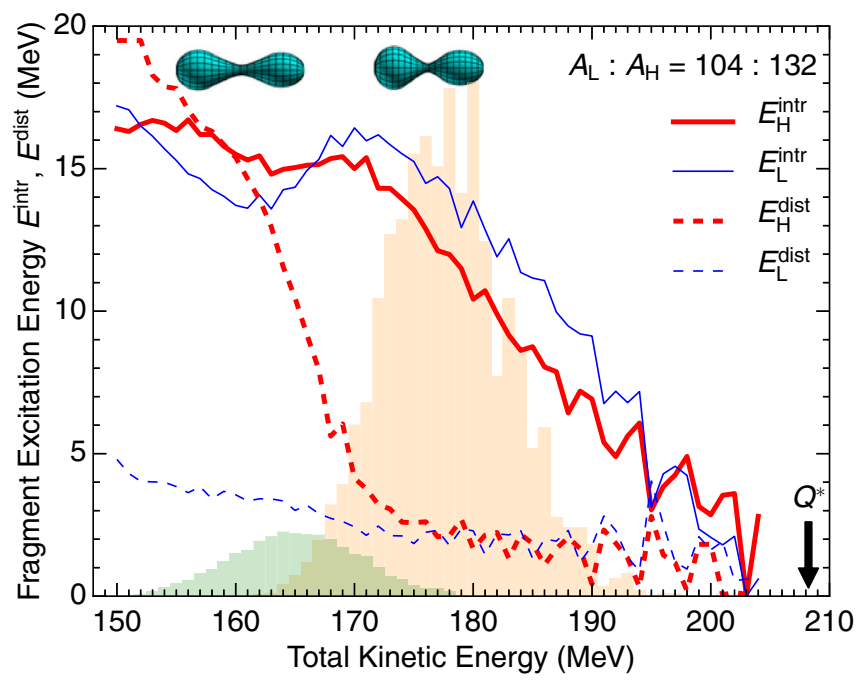

FIG. 11. Similar to Fig. 6(f), with fragment mass ratio $A_{\mathrm{L}}: A_{\mathrm{H}}=104: 132$, for $E_{n}=5.55 \mathrm{MeV}$. Probability densities are shown for the two modes: superlong (SL), with average shape specified by $q_{2}=16.5, \varepsilon_{\mathrm{L}}=0.15, \varepsilon_{\mathrm{H}}=0.32$ (green area), and standard (St) with average shape $q_{2}=11.5, \varepsilon_{\mathrm{L}}=0.25, \varepsilon_{\mathrm{H}}=-0.08$ (orange area). Typical scission shapes of the SL and St modes are also shown.

thermal fission for the two most symmetric cases. Such an evolution would be expected from the fact that the increased intrinsic excitation of the proto-fragments tends to wash out the structure effects that favored the light fragment. In the next section we discuss how the appearance of a new superlong fission mode plays an important role for this behavior.

\section{Bimodal fission}

Figure 11 shows the separate contributions to the final fragment excitation energy from their intrinsic and distortion energies at scission, displayed versus the resulting TKE for $A_{\mathrm{L}}: A_{\mathrm{H}}=104: 132$. As mentioned above, the increase in the kinetic energy of the incoming neutron primarily causes the intrinsic energy to increase and that in turn gives the system access to a wider variety of shapes during its evolution. This results in the appearance of a new fission mode characterized by more elongated scission shapes and, consequently, lower TKE values; see Fig. 3(b). On average, the scission shapes of these events have $q_{2} \approx 16.5$, significantly larger than those reached with thermal neutrons [Fig. 3(a)].

The existence of such a SL fission mode has long been known [23]. The mode favors symmetric fission, and it is believed that the observed increase of emitted neutrons near symmetry with incident neutron energy is caused by an increase in the yield of the superlong mode; see, e.g., Refs. [24,25].

For the large elongations characterizing the superlong mode it is preferable for the heavy fragment to develop a large quadrupole deformation and in average we have $\varepsilon_{\mathrm{H}}=0.32$. For small TKE values the average quadrupole moment of the heavy fragment is even larger, for example, $\varepsilon_{\mathrm{H}} \approx 0.5$ at TKE $=150 \mathrm{MeV}$. Also the light fragment is deformed but less so, $\varepsilon_{\mathrm{L}} \approx 0.15$. 
The scission shapes of the SL mode for $A_{\mathrm{L}}: A_{\mathrm{H}}=104: 132$ are compared in Fig. 3(b) with those obtained at higher TKE values where the elongation is much smaller, $q_{2} \approx 10-12$. In the standard (St) mode, the heavy fragment is slightly oblate, $\varepsilon_{\mathrm{H}} \approx-0.08$, while the light fragment has $\varepsilon_{\mathrm{L}} \approx 0.15$.

Figure 11 also shows calculated distributions of the two fission modes versus TKE. Guided by the result shown in Fig. 3(b), the SL mode is defined by the condition $q_{2}>14$ and correspondingly the St mode is defined by $q_{2}<14$. The St mode exhibits a broad distribution with its maximum at $\mathrm{TKE} \approx 180 \mathrm{MeV}$ and completely dominates the fission process at high TKE. But with decreasing TKE values the SL mode gradually appears, at first partly overlapping with the St mode but then taking completely over in the lowest TKE range.

The maximum of the SL distribution occurs at TKE $\approx$ $164 \mathrm{MeV}$. This average TKE value for the SL mode, converted from fragment to product kinetic energy, becomes $161 \mathrm{MeV}$, and is in reasonable agreement with the measured product kinetic energy for mass split $A_{\mathrm{L}}: A_{\mathrm{H}}=104: 132$, namely, $156 \mathrm{MeV}$ [26]. Also the calculated average TKE value for the St mode, $178 \mathrm{MeV}$, converted to the product kinetic energy $176 \mathrm{MeV}$, is in good agreement with the corresponding measured value, $174 \mathrm{MeV}$. Often [23,26], the St mode is described as consisting of two modes, $\mathrm{S} 1$ and $\mathrm{S} 2$, which together make up the asymmetric fission, and the value $174 \mathrm{MeV}$ is a weighted average over these modes from the results given in Ref. [26].

The large deformations of the heavy fragment in the SL mode imply very large distortion energies, $E_{\mathrm{H}}^{\text {dist }}=15-20 \mathrm{MeV}$ for TKE $=150-160 \mathrm{MeV}$, because the fragment shape has to relax from $\varepsilon_{\mathrm{H}}=0.4-0.5$ to its spherical ground-state shape. For comparison, the distortion energy of the heavy fragment in the St mode is only $2-3 \mathrm{MeV}$.

It is interesting to study how the partition of the intrinsic energy between the light and heavy fragments depends on the fission mode for mass division around $A_{\mathrm{L}}: A_{\mathrm{H}}=104: 132$. In the St mode the heavy fragment has a small oblate deformation, close to its doubly magic spherical ground state. The spherical shell gaps at $Z=50$ and $N=82$ lead to a very low level density for the heavy fragment, causing the light fragment to be favored; see Fig. 4. On the other hand, in the SL mode the heavy fragment has a substantial quadrupole deformation. The proto-fragment is thus far away from equilibrium and has a large single-particle level density near the Fermi level, leading to a large total level density. The distribution function for the intrinsic energy at scission, Eq. (4), then somewhat favors the heavy fragment.

Relative to the St mode, the heavy fragment receives significantly more excitation energy in the SL mode due to two different mechanisms: First, as just discussed, it is the favored recipient of intrinsic energy at scission. Second, as also mentioned above, it is very distorted at scission and its shape relaxation leads to an additional significant contribution.

The increase of the neutron multiplicity from the heavy fragment with increasing incident energy is thus partly due to the appearance of the SL mode. For thermal fission only the St mode appears and $\bar{\nu}_{\mathrm{L}}$ is larger than $\bar{\nu}_{\mathrm{H}}$ for all TKE. With increasing neutron energy the SL mode appears and causes

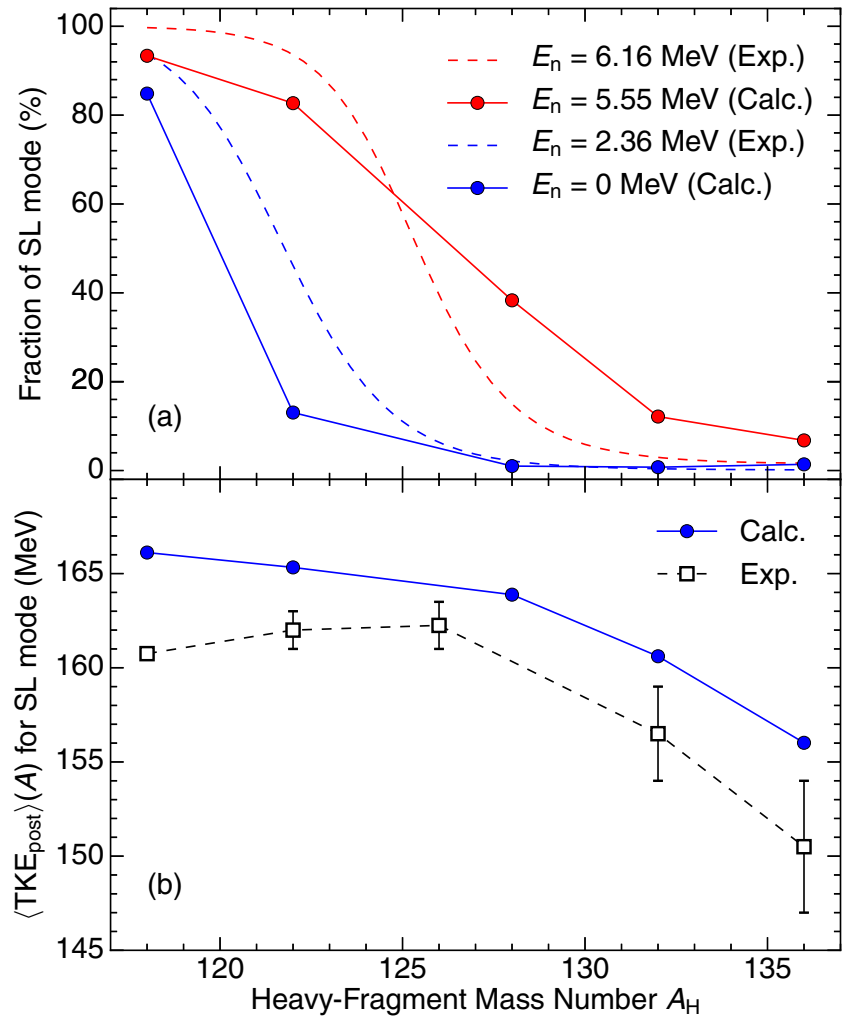

FIG. 12. (a) The relative weight of the SL mode vs the heavyfragment mass number $A_{\mathrm{H}}$ either calculated for $E_{n}=0$ and $5.55 \mathrm{MeV}$ or measured [26] for $E_{n}=2.36$ and 6.14 MeV. (b) Average TKE $E_{\text {post }}$ vs $A_{\mathrm{H}}$ for the SL mode either calculated for $E_{n}=5.55 \mathrm{MeV}$ or measured [26]. The calculated TKE energies are converted from fragment to product kinetic energies (post).

the heavy fragment to become preferentially more excited. Consequently, with increasing $E_{n}, \bar{v}_{\mathrm{H}}$ increases faster than $\bar{\nu}_{\mathrm{L}}$.

Indeed, it has been found experimentally [14] that the additional prompt neutrons emitted when the incident neutron energy is increased originate mainly from the heavy fragment. The onset of the SL mode with increasing neutron energy provides an additional mechanism for the neutron multiplicity from the heavy fragment to increase more than from the light fragment.

\section{The superlong mode in near-symmetric fission}

After the above detailed analysis of the role of the SL mode for the mass division $A_{\mathrm{L}}: A_{\mathrm{H}}=104: 132$, we now study its role in a broader region around symmetry where it is most prominent. But we note that it grows increasingly significant also for more asymmetric fission as the incoming neutron energy is raised.

The main features are apparent from Figs. 8 and 10. For thermal fission (Fig. 8) the SL mode plays no role for divisions with $A_{\mathrm{H}} \geqslant 128$, appearing only for the most symmetric division shown, $A_{\mathrm{H}}=122$, but for $E_{n}=5.55 \mathrm{MeV}$ (Fig. 10) it has become the dominant mode for $A_{\mathrm{H}}=122$ and it remains visible up to $A_{\mathrm{H}}=136$.

A more complete view can be gained from Fig. 12(a) where the mass dependence of the relative prominence of the SL 
mode is shown for various incident energies. The SL fractions calculated for $E_{n}=0$ and $5.55 \mathrm{MeV}$ are shown together with values measured for $E_{n}=2.36$ and $6.14 \mathrm{MeV}$ [26]. The agreement between theory and experiment is reasonably good.

The mass dependence of the average TKE for the SL fission mode calculated for $E_{n}=5.55 \mathrm{MeV}$ is shown in Fig. 12(b) together with data taken from the analysis of Ref. [26], assumed for neutron energies in a broad region. The two curves are rather similar, but the measured values are systematically 3-5 MeV lower.

It is apparent from Figs. 8 and 10 that the two fission modes have significantly different TKE distributions, with the SL distribution being centered 10-15 MeV below the St distribution. Because the relative weight of those two distribution depends on the mass division, the fission observables should exhibit corresponding evolutions. One signature of this bimodality is the sudden change in the average TKE seen in Fig. 1(b) around $A_{\mathrm{H}}=130$ where the dominant mode switches. Another typical bimodal signature is the maximum in the TKE fluctuations seen in Fig. 1(d) around $A_{\mathrm{H}}=128$ where the two modes have similar weights.

\section{SUMMARY AND DISCUSSION}

Using macroscopic-microscopic potential-energy surfaces in the 3QS shape parametrization and microscopic level densities, we have applied the METROPOLIS random-walk method to the induced fission reaction ${ }^{235} \mathrm{U}(n, f)$ at $E_{n}=0$ and 5.55 MeV. Because the calculational method generates an ensemble of individual fission events it is possible to extract a large variety of correlations. We have particularly studied $\bar{v}(A ; \mathrm{TKE})$, the average neutron multiplicity as function of the primary fission-fragment mass number $A$ for events leading to a specified total fragment kinetic energy TKE. For a given mass division, $A_{0} \rightarrow A_{\mathrm{L}}+A_{\mathrm{H}}$, the constraint on TKE restricts the total combined fragment excitation energy to be $\mathrm{TXE}=Q_{\mathrm{LH}}^{*}-\mathrm{TKE}$. The excitation of each fragment is composed of two terms: its share of the total available intrinsic excitation energy at scission and the energy recovered from the relaxation of its distorted shape at scission. The division of the intrinsic excitation energy between the two distorted proto-fragments is carried out statistically based on their microscopic level densities. As a consequence, the contributions to each fragment from the intrinsic excitation energy and the distortion energy vary with $A$ and TKE in a nontrivial manner.

An indirect observation of the excitation energy in each primary fragment can be made through the mass-dependent neutron multiplicity, $\bar{v}(A)$. For thermal fission, where TKEgated data are available, a good agreement is obtained between the calculated and the measured TKE-gated multiplicities, $\bar{v}(A ; \mathrm{TKE})$. This agreement extends to several finer details, such as the threshold effect in the neutron multiplicity from the light fragment with decreasing TKE in fission leading to the division $A_{\mathrm{L}}: A_{\mathrm{H}}=82: 154$. Certain differences between the calculated and the measured $\bar{v}(A ; \mathrm{TKE})$ for $A_{\mathrm{L}}: A_{\mathrm{H}}=104: 132$ may be due to the fact that the calculation assumes that all the fragments have the same $N / Z$ ratio. The inclusion of the fragment isospin degree of freedom would probably make ${ }^{132} \mathrm{Sn}$ the most favored fragment for $A=132$, rather than the neighboring ${ }^{132} \mathrm{Te}$, and, consequently, would increase the difference between $\bar{v}\left(A_{\mathrm{L}}\right.$; TKE $)$ and $\bar{v}\left(A_{\mathrm{H}}\right.$; TKE $)$, as is experimentally observed.

For the near-symmetric mass division $A_{\mathrm{L}}: A_{\mathrm{H}}=108: 128$ the deviations between data and calculated TKE-gated neutron multiplicity are substantial. The observed very small increase in the heavy-fragment neutron multiplicity as TKE is decreased could not be reproduced. However, generating the same change in average excitation energy by use of energetic incident neutrons yields a much larger increase in neutron multiplicity [14], in good agreement with the calculations.

We also studied $\bar{v}(A ; \mathrm{TXE})$, the mass-dependent average neutron multiplicity for events having the specified value of TXE, the combined excitation energy of the two primary fragments. This allows more detailed studies of the sawtooth behavior of $\bar{v}(A)$ with excitation energy.

An increase of the incident neutron energy to $E_{n}=$ $5.55 \mathrm{MeV}$ leads to a more prominent role of a new superlong fission mode characterized by low TKE values and occurring preferentially in the near-symmetric region, in agreement with experimental findings. In the SL fission events, which involve very elongated scission configurations, the heavy proto-fragment is particularly distorted, implying a large distortion energy, but also a high level density. For these reasons, most of the additional energy brought in by the neutron goes to excitation of the heavy primary fragment. Consequently, in the near-symmetric region, $\bar{\nu}_{\mathrm{H}}$ increases faster than $\bar{\nu}_{\mathrm{L}}$ with increasing $E_{n}$, as is also observed experimentally.

The dependence of the relative fraction of the SL mode on the fragment mass was found to agree well with data. Also the average TKE value associated with SL fission events was found to be in good agreement with data.

At $E_{n}=5.55 \mathrm{MeV}$, the SL mode is visible for the mass divisions $A_{\mathrm{L}}: A_{\mathrm{H}}=104: 132,100: 136,108: 128$, and 114:122 and, with decreasing TKE, $\left(\bar{v}_{\mathrm{L}} ; \mathrm{TKE}\right)$ and $\left(\bar{\nu}_{\mathrm{H}}\right.$; TKE) cross so the heavy fragment becomes the dominant neutron emitter at low TKE [Figs. 10(e) and 10(f)]. This prediction could be tested experimentally with TKE-gated multiplicity data obtained for higher incident energies.

The results in the symmetric fission region are less accurate in our present calculations because the scission configurations encountered for near-symmetric divisions are insufficiently elongated and hence lead to too large TKE values. This may be due to limitations in the employed 3QS shape parametrization and we are currently investigating whether a suitable extension can be developed.

Other models of the fission process, such as those employed in Refs. [3-6], suggest larger distortions of the proto-fragments than what is obtained in the present treatment. Those models may nevertheless be able to also give a reasonable reproduction of the measured neutron multiplicities if they compensate for the large distortion energies by giving correspondingly smaller intrinsic energies. In order to elucidate the situation, it would be very valuable to calculate within those models as well the TKE-gated neutron multiplicities, $\bar{v}(A ; \mathrm{TKE})$. Detailed comparisons of this observable, both between the various models and with the experimental data, might reveal the quantitative importance of the different contributions to the fragment excitations 
and thus help to improve our understanding of the fission process.

\section{ACKNOWLEDGMENTS}

We acknowledge discussions with C. Schmidt, and thank A. Göök and W. Loveland for providing information about the experimental data. Comments on the paper by R. Vogt are acknowledged. This work was supported by the Swedish Natural Science Research Council (S.A.) and the Knut and Alice Wallenberg Foundation (M.A., B.G.C., and S.Å.); J.R. was supported in part by the Office of Nuclear Physics in the U.S. Department of Energy's Office of Science under Contract No. DE-AC02-05CH11231.
[1] K. N. Y. Nakagome, H. Yamamoto, and I. Kimura, Nucl. Phys. A 632, 540 (1998).

[2] B. D. Wilkins, E. P. Steinberg, and R. R. Chasman, Phys. Rev. C 14, 1832 (1976).

[3] N. Dubray, H. Goutte, and J.-P. Delaroche, Phys. Rev. C 77, 014310 (2008).

[4] G. Scamps and C. Simenel, Nature (London) 564, 382 (2018).

[5] A. Bulgac, P. Magierski, K. J. Roche, and I. Stetcu, Phys. Rev. Lett. 116, 122504 (2016); A. Bulgac, S. Jin, and I. Stetcu, Phys. Rev. C 100, 014615 (2019).

[6] A. Bulgac, S. Jin, K. J. Roche, N. Schunck, and I. Stetcu, Phys. Rev. C 100, 034615 (2019).

[7] M. Albertsson, B. G. Carlsson, T. Døssing, P. Möller, J. Randrup, and S. Åberg, Phys. Lett. B 903, 135276 (2020).

[8] A. Göök, F.-J. Hambsch, S. Oberstedt, and M. Vidali, Phys. Rev. C 98, 044615 (2018); A. Göök (private communication).

[9] J. Randrup and P. Möller, Phys. Rev. Lett. 106, 132503 (2011).

[10] J. Randrup, P. Möller, and A. J. Sierk, Phys. Rev. C 84, 034613 (2011).

[11] J. Randrup and P. Möller, Phys. Rev. C 88, 064606 (2013).

[12] H. Uhrenholt, S. Åberg, A. Dobrowolski, T. Døssing, T. Ichikawa, and P. Möller, Nucl. Phys. A 913, 127 (2013).
[13] D. E. Ward, B. G. Carlsson, T. Døssing, P. Möller, J. Randrup, and S. Åberg, Phys. Rev. C 95, 024618 (2017).

[14] R. Müller, A. A. Naqvi, F. Käppeler, and F. Dickmann, Phys. Rev. C 29, 885 (1984).

[15] P. Möller, A. J. Sierk, T. Ichikawa, A. Iwamoto, R. Bengtsson, H. Uhrenholt, and S. Åberg, Phys. Rev. C 79, 064304 (2009).

[16] J. R. Nix, Nucl. Phys. A 130, 241 (1969).

[17] A. Tudora, Eur. Phys. J. A 55, 98 (2019).

[18] A. Al-Adili, Ph.D. thesis, Uppsala University, 2013, p. 51; A. Oberstedt (private communication).

[19] M. D. Usang, F. A. Ivanyuk, C. Ishizuka, and S. Chiba, Sci. Rep. 9, 1525 (2019).

[20] J. Randrup and R. Vogt, Phys. Rev. C 80, 024601 (2009).

[21] P. Möller and T. Ichikawa, Eur. Phys. J. A 52, 173 (2015).

[22] M. B. Chadwick et al., Nucl. Data Sheets 148, 189 (2018).

[23] U. Brosa, S. Grossmann, and A. M. Müller, Phys. Rep. 197, 167 (1990).

[24] F.-J. Hambsch, S. Oberstedt, A. Tudora, G. Vladuca, and I. Ruskov, Nucl. Phys. A 726, 248 (2003).

[25] K. H. Schmidt and B. Jurado, Phys. Rev. Lett. 104, 212501 (2010).

[26] R. Yanez, W. Loveland, J. King, J. S. Barrett, N. Fotiades, and H. Y. Lee, arXiv:1605.09690v4. 